\title{
The Satisfaction Level of Patients Seeking Dental Care in the Slovak Republic: A Cross-sectional Questionnaire Study (Original paper)
}

\section{Samohyl (Martin Samohyl)', A. Nadazdyova (Anna Nadazdyova)², M. Hirjak (Martin Hirjak)33, L. Argalasova (Lubica Argalasova)', K. Hirosova (Katarina Hirosova)', J. Jurkovicova (Jana Jurkovicova) ${ }^{1}$}

${ }^{1}$ Institute of Hygiene, Faculty of Medicine,

2 Department of Stomatology and Maxilofacial Surgery, Faculty of Medicine, Comenius University in Bratislava, Slovakia

${ }^{3}$ Department of Public Health, St. Elizabeth's College of Health and Social Sciences, Bratislava, Slovakia

\section{E-mail address:}

martin.samohyl@fmed.uniba.sk

\section{Reprint address:}

Martin Samohyl

Comenius University in Bratislava

School of medicine

Bratislava, Slovakia

Suource: Clinical Social Work and Health Intervention Pages: $34-42$
Volume: 8

Cited references: 22

Issue: 3

\section{Reviewers:}

Johnson Mawole

Department of Sociology and Statistics, University of Mwanza, Tanzania

George Herdics

School of Management Warsaw University of Management, Poland

\section{Key words:}

PSQ III, satisfaction, dental care.

\section{Publisher:}

International Society of Applied Preventive Medicine i-gap 


\section{Abstract:}

Objective: To determine the level of the healthcare quality satisfaction according to some demographic variables (gender, age, education level, employment); change/no change of Dentist during the past year; extra payment for the healthcare; the distance to the Dentist.

Design: A Cross-sectional Questionnaire Study.

Participants: Completed questionnaires were obtained from 433 subjects (53.6\% men, $46.4 \%$ of women, mean age $35.9 \pm 14.6$ years).

Methods: A standardized Patient Satisfaction Questionnaire (PSQ III, long-form) was used.

Results: General healthcare quality satisfaction was identical (or nearly identical) in subgroups according to gender, changing Dentist, and extra payment for healthcare. The age and extra payment for healthcare had no impact on the assessment in any subscale. The overall satisfaction rate suggested significant differences within the subgroups according to gender, education level, and changing Dentist.

Conclusion: Satisfaction of dental patients was observed to be different in various demographic subgroups and across several subscales of satisfaction assessment.

\section{Introduction}

Football is a popular outdoor activity often used as a social intervention in refugee camps to prevent cabin fever and promote healthy social and physical interactions. However, sportsmen are at risk of acquiring SSTI usually caused by $S$. aureus. Wet towels, close contact at a match, physiotherapeutic procedures and massages are risk factors. The aim of this study was to assess the proportion of MRSA among football players in the refugee camp at Veria, Greece, which serves about 5,000-7,000 migrants waiting for asylum procedures.

\section{Methods}

In this cross-sectional study, the standardized Patient Satisfaction Questionnaire PSQ III (long-form) was used(5). The questionnaire itself was preceded by several questions regarding basic demographic data; questions about the reasons for changing Dentists during the past year; extra payment for healthcare; distance to the Dentist. The standardized questionnaire $P S Q I I I$ consists of 50 statements focusing on the quality of the healthcare provided. Respondents were free to choose an answer on a scale from 1 to 5 - to what extent they agree with each statement: (1 strongly agree, 2 agree, 3 not sure, 4 disagree, 5 strongly disagree).

The statements in the standardized questionnaire are divided into seven subscales:

- General satisfaction (6 questions); technical quality (10 questions);

- Interpersonal aspects (7 questions); communication (5 questions);

- Financial aspects (8 questions); time spent with the Dentist (2 questions); Access/availability/convenience (12 questions);

- We evaluated the overall satisfaction (50 questions altogether) as well.

Answers to some questions where strong agreement means the maximum satisfaction with the HCQ had to be rescaled (strongly 
agree 5, agree 4 disagree 2, strongly disagree 1) to obtain a unified HCQ score: 1 $=$ maximum dissatisfaction with the $\mathrm{HCQ}$, $5=$ maximum satisfaction with the HCQ. Particular groups of questions reflecting the degree of HCQ satisfaction were evaluated as mean scores for each subscale. The highest average value means the highest level of HCQ satisfaction.

The study sample was recruited from patients attending dental surgeries in the capital of Slovakia and in several small towns in western and eastern regions of Slovakia; questionnaires were collected from November 2014 to April 2015. In total, 433 completed questionnaires were collected; the response rate was 93\%. Parents completed a questionnaire instead of underage respondents. The questionnaire was anonymous and a privacy policy was respected; participation in the study was voluntary.

The whole sample was divided into subgroups according to gender; age; education level; employment; change/no change of Dentist within the last year; extra payment for the Healthcare; the distance to the Dentist. The sample consisted of 433 respondents $(53.6 \%$ of men, $46.4 \%$ of women) aged 15-75 years; mean age was $35.9 \pm 14.6$ years. The majority of respondents were in the age group $30-49$ years $(41.6 \%)$. In terms of employment, most subjects were classified as employed workers $(61.4 \%)$; students (22.4\%); pensioners (7.8\%). Subjects with a secondary education represented the largest group (64.3\%), followed by subjects with a University (25.1\%) and elementary education $(9.7 \%)$.

Data was analyzed using the statistical program SPSS. Descriptive statistics (percentages, averages, standard deviations) were used. A two-sample T-test was used to compare the mean scores of HCQ satisfaction in subgroups according to gender; changing Dentist; the amount of extra payment for healthcare; distance to the Dentist. An ANOVA test was used to compare the mean scores of HCQ satisfaction in subgroups according to age, occupation and education level. The statistically significant level was determined at $P$ values $<0.05$.

\section{Results}

Mean scores in all subscales of HCQ satisfaction are presented in Table 1. General HCQ satisfaction was identical (or nearly identical) in subgroups according to gender; changing Dentist; extra payment for healthcare. A higher, although not significant satisfaction level was noticed in the oldest patients; in those with the lowest education level; and in pensioners. Lowest general satisfaction was declared by unemployed patients $(2.36 \pm 0.24)$ and patients who have to commute long distances to the Dentist $(2.34 \pm 0.25)$. The only statistically significant difference was found between subgroups according to distance to the Dentist $(P=0.013)$.

Men were significantly less satisfied with technical aspect of HCQ compared to women $(2.55 \pm 0.25$ and $2.62 \pm 0.26$, respectively; $P=0.008)$. Age, extra payment for healthcare, and distance to the Dentist had almost no impact to the assessment of technical aspect of HCQ. The most satisfied subgroups were patients with a secondary education level, students, and patients who had changed their Dentist (the differences in scoring in subgroups according to occupation and changing Dentist were significant ( $P=0.014$ and $P=0.005$, respectively). 


\begin{tabular}{|c|c|c|c|c|c|c|c|c|c|c|c|c|c|}
\hline \multirow{2}{*}{ 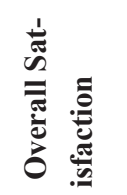 } & \multirow{2}{*}{$\begin{array}{l}a \\
* \widehat{\theta}\end{array}$} & & \multicolumn{2}{|r|}{ तิ } & & \multicolumn{3}{|c|}{ 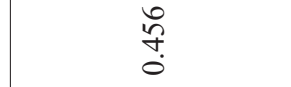 } & & \multicolumn{4}{|c|}{ ठิ. } \\
\hline & & & 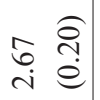 & $\begin{array}{lc}\stackrel{\overbrace{}}{i} \\
\stackrel{i}{e}\end{array}$ & & $\begin{array}{ll}\infty & \hat{\sigma} \\
\infty & \overrightarrow{0} \\
i & 0\end{array}$ & 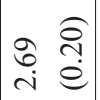 & 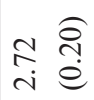 & & 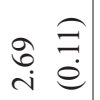 & 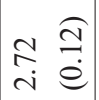 & $\begin{array}{ll}\stackrel{\infty}{r} & \frac{0}{e} \\
i & \end{array}$ & 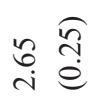 \\
\hline 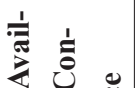 & 2 & & \multicolumn{2}{|r|}{$\frac{\text { I }}{\stackrel{+}{0}}$} & \multicolumn{4}{|c|}{ 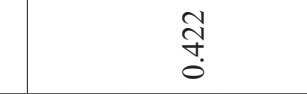 } & & \multicolumn{4}{|c|}{ 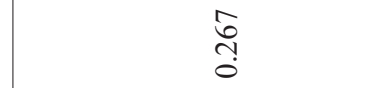 } \\
\hline 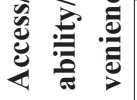 & $\star \widehat{\hat{\theta}}$ & & 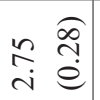 & 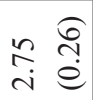 & & $\begin{array}{cc}m & \bar{m} \\
\stackrel{n}{i} & e\end{array}$ & 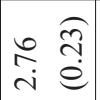 & \begin{tabular}{ll}
$\infty$ & $\widehat{\hat{\jmath}}$ \\
\multirow{2}{*}{} & $\hat{e}$
\end{tabular} & & 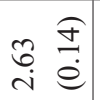 & $\begin{array}{ll}\stackrel{\sigma}{a} \\
\stackrel{a}{e}\end{array}$ & \begin{tabular}{ll}
\multirow{\tau}{*}{} & $\widehat{ก}$ \\
$\dot{\imath}$ & $\hat{e}$
\end{tabular} & 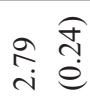 \\
\hline \multirow{2}{*}{ 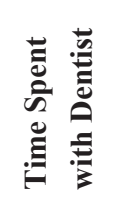 } & 2 & & \multicolumn{2}{|r|}{$\frac{n}{0}$} & \multicolumn{4}{|c|}{$\stackrel{0}{=}$} & & \multicolumn{4}{|c|}{$\stackrel{n}{\tilde{n}}$} \\
\hline & $\star \widehat{\hat{\theta}}$ & & $\begin{array}{ll}+ & 0 \\
i & 0 \\
i & e\end{array}$ & 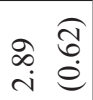 & & 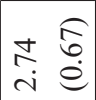 & $\begin{array}{ll}\infty & \widehat{\sigma} \\
\infty & 0 \\
i & e\end{array}$ & $\begin{array}{ll}\infty & \tilde{n} \\
\infty & n \\
i & \stackrel{e}{e}\end{array}$ & & $\begin{array}{ll}n & \widehat{o} \\
& \stackrel{n}{e} \\
& 0\end{array}$ & 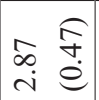 & $\begin{array}{ll} & \widehat{n} \\
\infty & n \\
i & e\end{array}$ & $\begin{array}{ll} & \hat{0} \\
\stackrel{0}{0} & \infty \\
i & 0\end{array}$ \\
\hline \multirow{2}{*}{ 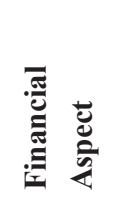 } & 2 & & \multicolumn{2}{|r|}{$c$} & \multicolumn{4}{|c|}{$\begin{array}{l}\infty \\
\infty \\
0 \\
0\end{array}$} & & \multicolumn{4}{|c|}{$\stackrel{\widetilde{N}}{\tilde{0}}$} \\
\hline & $\triangle \widehat{\hat{\theta}}$ & & 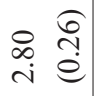 & 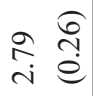 & & $\begin{array}{ll}\overrightarrow{\hat{\sigma}} \\
\dot{\infty} & \tilde{e}\end{array}$ & 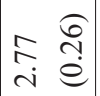 & 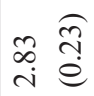 & & 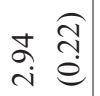 & $\begin{array}{ll}\sim & \widehat{\sigma} \\
\infty & \vec{e} \\
\sim & 0\end{array}$ & 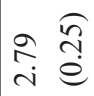 & 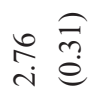 \\
\hline \multirow{2}{*}{ : } & 2 & & \multicolumn{2}{|r|}{$\begin{array}{l}\mathscr{\infty} \\
\infty \\
0\end{array}$} & \multicolumn{4}{|c|}{ 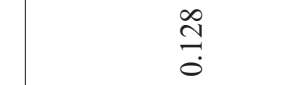 } & & \multicolumn{4}{|c|}{$\overrightarrow{8}$} \\
\hline & $\star \widehat{\hat{\theta}}$ & & $\begin{array}{ll}\vec{\sigma} & \widehat{\eta} \\
\dot{\sigma} & \hat{e}\end{array}$ & 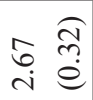 & & $\begin{array}{ll} & \hat{\theta} \\
\overrightarrow{0} & ? \\
i & 0 \\
i & 0\end{array}$ & 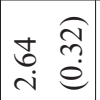 & 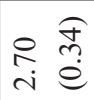 & & 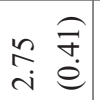 & $\begin{array}{cc}0 & \bar{m} \\
i & \tilde{e} \\
i & e\end{array}$ & 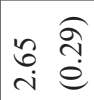 & 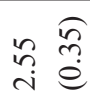 \\
\hline \multirow{2}{*}{ 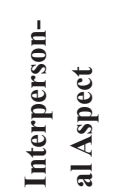 } & $a$ & & & \multicolumn{4}{|c|}{$\frac{\infty}{0}$} & & \multicolumn{4}{|c|}{$\stackrel{n}{+}$} \\
\hline & 4 氙 & & 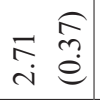 & 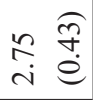 & & 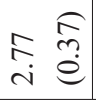 & $\begin{array}{ll}\hat{\sigma} & \widehat{\nabla} \\
i & \stackrel{\theta}{e}\end{array}$ & $\begin{array}{ll}\stackrel{f}{f} \\
\stackrel{i}{i} & \stackrel{e}{e}\end{array}$ & & $\begin{array}{ll} & 0 \\
\sigma & 0 \\
ن & e \\
ن & e\end{array}$ & $\begin{array}{ll}\sim & \hat{\jmath} \\
i & \hat{e}\end{array}$ & $\begin{array}{ll}\circ & \hat{q} \\
\infty & \dot{+} \\
i & \dot{e}\end{array}$ & $\begin{array}{ll}\infty & \widehat{\vec{n}} \\
0 & 0 \\
i & e\end{array}$ \\
\hline \multirow{2}{*}{ 冚 } & 2 & & & \multicolumn{4}{|c|}{ ڤn } & & \multicolumn{4}{|c|}{$\ddot{\circ}$} \\
\hline & $\star \widehat{\hat{\theta}}$ & & 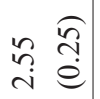 & 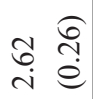 & & 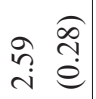 & 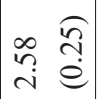 & 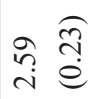 & & $\begin{array}{ll} & \widehat{\theta} \\
\stackrel{\vartheta}{*} & \vdots \\
ن & e\end{array}$ & $\begin{array}{cc}0 & \infty \\
\sim & \vec{e} \\
\sim & 0\end{array}$ & 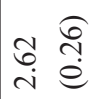 & $\stackrel{\substack{\infty\\
}}{\stackrel{c}{e}}$ \\
\hline \multirow{2}{*}{ 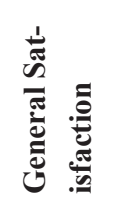 } & $a$ & & \multicolumn{2}{|c|}{$\begin{array}{l}0 \\
\infty \\
\infty \\
0\end{array}$} & \multicolumn{4}{|c|}{$\stackrel{0}{\stackrel{0}{0}}$} & & \multicolumn{4}{|c|}{ ڤั } \\
\hline & ब氙 & & 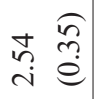 & 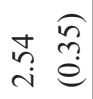 & & 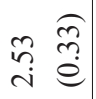 & $\begin{array}{cc}\sim & \hat{n} \\
\sim & 0 \\
\sim & 0\end{array}$ & $\begin{array}{ll}\vec{n} & \tilde{\sigma} \\
i & 0\end{array}$ & & 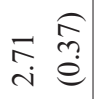 & $\begin{array}{ll}\bar{\sigma} & \widehat{\tilde{n}} \\
i & \hat{e}\end{array}$ & 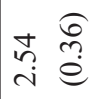 & $\vec{n} \tilde{n}$ \\
\hline & & $\underbrace{e}_{a}$ & $\begin{array}{ll} & \widehat{0} \\
\approx & \dot{n} \\
\approx & 0\end{array}$ & 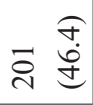 & & $\begin{array}{ll} & 6 \\
& \dot{9} \\
= & \end{array}$ & 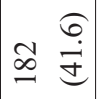 & $\cong \stackrel{\infty}{\Xi}$ & & $+\hat{e}$ & भ & 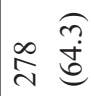 & g) \\
\hline & $\frac{0}{\frac{0}{a}}$ & : & $\frac{0}{\sigma}$ & $\begin{array}{l}\frac{0}{\pi} \\
\text { है } \\
\text { II }\end{array}$ & 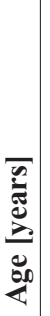 & 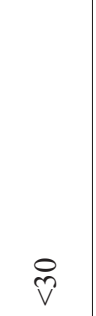 & $\begin{array}{l}\text { qे } \\
\text { oे }\end{array}$ & 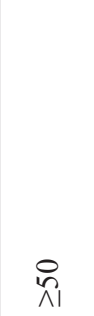 & 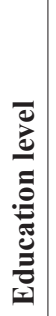 & 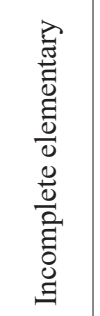 & 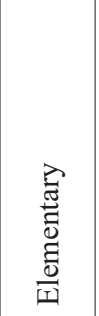 & 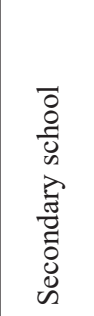 & 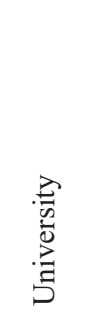 \\
\hline
\end{tabular}




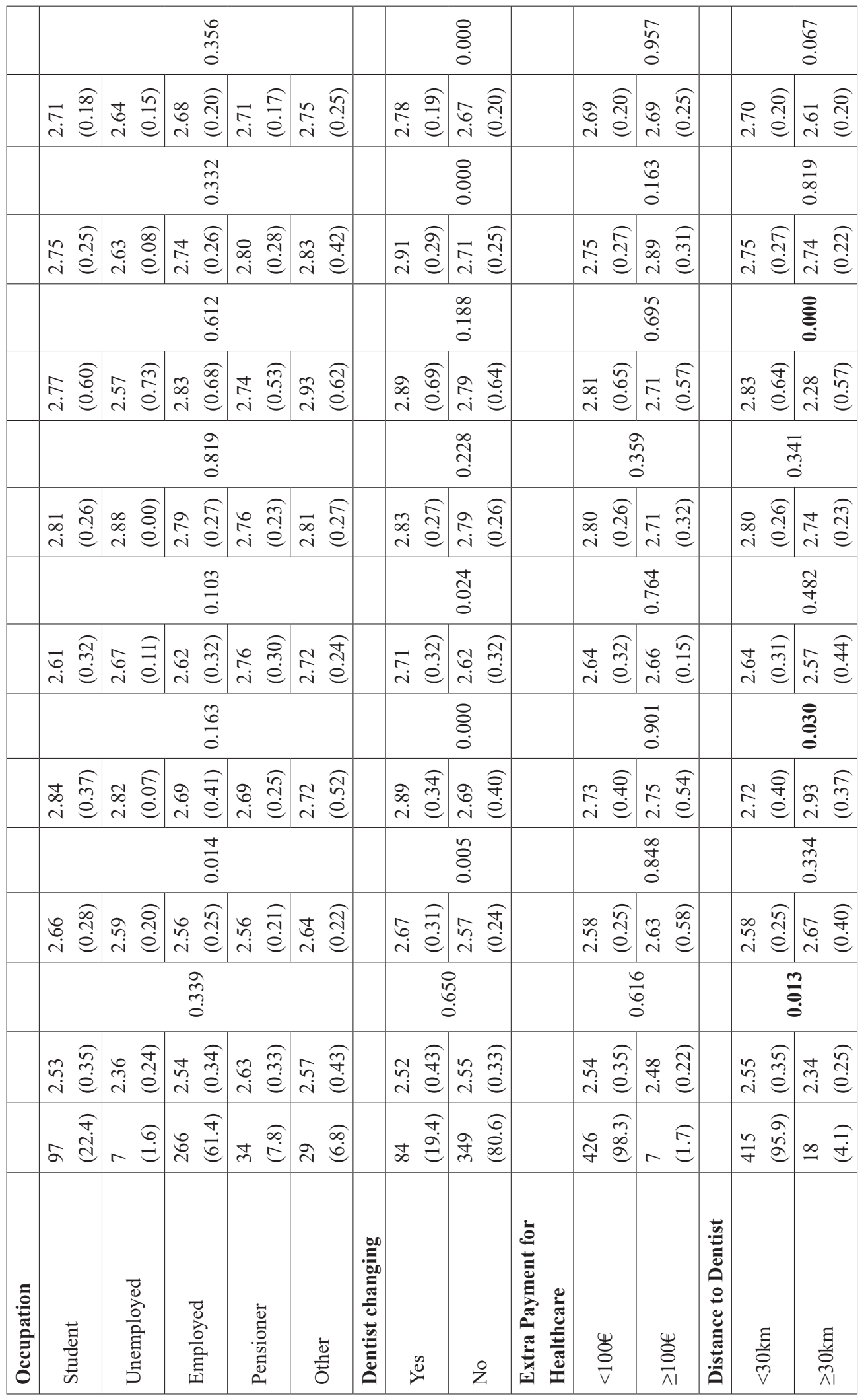


The significant differences in the interpersonal aspect subscale were found in subgroups according to changing Dentist $(P=0.000)$ and distance to the Dentist $(P=0.030)$; more satisfied were patients who had changed their Dentist and those with longer distance to the Dentist. Gender, age, education level, occupation and extra payment had no significant impact on the HCQ assessment in this subscale.

In the subscale communication with the Dentist, the highest level of satisfaction was observed in the oldest age group $(2.70 \pm 0.34)$; in patients with elementary education level $(2.76 \pm 0.31)$; in pensioners $(2.76 \pm 0.30)$; in Dentist changing patients $(2.71 \pm 0.32)$. Significant differences in this subscale were found in subgroups according to education level $(P=0.001)$ and changing Dentist $(P=0.024)$.

We did not find any significant differences among subgroups in the subscale financial aspect of HCQ satisfaction. The highest level of dissatisfaction in this subscale was observed in the age group 30-49 years $(2.77 \pm 0.26)$; in patients with University education $(2.76 \pm 0.31)$; in pensioners $(2.76 \pm 0.23)$; in patients with a higher extra payment for healthcare $(2.71 \pm 0.32)$; and in those with long distances to the Dentist (2.74 \pm 0.23$)$.

Women were significantly more satisfied with HCQ in the subscale time spent with the Dentist than men $(2.89 \pm 0.62$ and $2.74 \pm 0.66$, respectively; $P=0.015)$. The high significant difference was observed between patients subgroups according to distance to the Dentist: in this subscale, the most dissatisfied patients were those with a longer distance to the Dentist $(2.28 \pm 0.57 ; P=0.000)$.

The high significant difference in the subscale access/availability/convenience was achieved in the variable Dentist changing: the highest level of satisfaction in this subscale was declared by patients who had changed their Dentist in the last year $(2.91 \pm 0.29$ vs. $2.71 \pm 0.25 ; P=0.000)$.

The overall HCQ satisfaction assessment suggested significant differences within subgroups according to gender $(P=0.022)$; education level $(P=0.029)$; changing Dentist $(P=0.000)$. Higher levels of overall dissatisfaction were declared by men $(2.67 \pm 0.20)$; the youngest age group (2.68 \pm 0.19$)$; patients with university education $(2.65 \pm 0.25)$; unemployed subjects $(2.64 \pm 0.15)$; patients who had not changed their Dentist within the last year $(2.67 \pm 0.20)$; those with long distances to the Dentist (2.61 \pm 0.20$)$.

\section{Discussion}

General patient satisfaction is subjective, because patients do not take into account the appropriateness and results of therapy(6). The mean general satisfaction in our study was lower than reported by Chander et al.(7), Holikatti et al.(8). This can be partly explained by the fact that the studies mentioned above dealt with different patient samples and used the short-form questionnaire PSQ-18 (only 3 general satisfaction questions); whereas in our study the longform questionnaire PSQ III (6 general satisfaction questions) was used.

An average score of patient satisfaction in the subscale technical quality is in our study nearly the same as in the study by Holikatti et al.(8); the highest level of satisfaction was declared by Dentist changing patients and patients who commute long distance to their Dentist. Technical quality is based upon objective criteria; satisfaction is subjective. Satisfaction reflects both the patient's subjective assessment of quality of care and expectations for it. Edlund et 
al.(9) confirmed the appropriate technical quality of healthcare leading to greater patient satisfaction. However, many aspects of technical quality of healthcare should not be evaluated by patients. Dentists who provide a high level of technical quality might not be highly rated by patients(10). Dentists in small towns and villages in Slovakia usually lack higher standard equipment such as panoramic X-ray devices, and this fact forces patients to commute long distances to a center with the required medical equipment.

The quality of communication between Dentists and patients is a multidimensional concept that includes medical technology, psychosocial aspects and interaction(11). Proper communication skills have often been neglected in medical education in the EU as well as in the USA(12). An important feature of communication processes is the fact that they can convey messages through multiple layers of verbal and nonverbal communication, and may indirectly facilitate the analysis of healthcare(13). Our study showed far lower rate in the subscale communication compared with Jagadeesan's study who reported the mean score in ophthalmological patients in this subscale 4.46(14). We found the highest mean scores in the subscale communication among pensioners as well as among patients with elementary education level (deemed to be of less frequent users of the Internet). Current knowledge about Internet users seems to be limited concerning the number and demographic profile of people acquainted with this medium(15). Currently, when communicating with a Dentist, the Internet is the preferred medium. A direct link between Internet use and HCQ rates is confirmed by Esmaeilzadeh et al.(16). Errors in communication between healthcare professionals and patients can lead to medical errors which have unintended consequences for a patient's condition(17). The approach concentrated on communication between health-care professionals and patients is considered a valuable strategy in creating relationships with patients, and is considered a key aspect of HCQ improvement(18).

Health cost must be included in economic calculations of all sectors and at all levels of governance. Some answers in this subscale could be skewed because the kind of medical examination was not taken into account. HCQ in this subscale was assessed as poor when compared with studies by Nordyk et al. (19), Ziaei et al.(20). However, patient mean age in Nordyk's study was higher than in our study. In general, satisfaction appears to be higher in older patients(21). This fact was partly confirmed by our study, too.

Patient satisfaction found in the subscale time spent with Dentist was lower than in studies by Ziaei et al.(20), Chander et al.(7), Holikatti et al.(8). However, in our sample patients declared the highest level of satisfaction right in this subscale. Dentist should not only be focused on finishing the treatment as fast as possible just to minimize the waiting time, but also should concentrate on explaining the treatment as well as the treatment options, to patient's satisfaction(22).

Access to health services is a prerequisite for a high HCQ. Simultaneously, access to health-care services is considered as one of the cornerstones of the HCQ, and an important strategy for providing healthcare for all. Healthcare should be available without restrictions to every citizen near the place where he/she lives.

\section{Conclusion}

In conclusion, patient satisfaction is a complex issue with various influencing factors. Overall satisfaction with the dental 
service was observed to be varied across demographic groups: men; youngest patients; unemployed; patients with University education were least satisfied.

Our result suggests a series of recommendations:

- Create training programs for Dentists to can improve their communication skills.

- Promote the development of a team that will support the implementation of changes in the HCQ at the national level. This approach would be based on the knowledge of HCQ Theory and would strive to achieve positive changes in healthcare facilities as well.

- Introduce mentorship (i.e. the way employees are managed) as an additional tool for HCQ improvement.

\section{Reference}

1. DUBOVA SVD, FLORES - HERNANDEZ S, RODRIGUEZ - AGUILAR L, PÉREZ - CUEVAS R (2010) Quality of care and health-related quality of life of climacteric stage women cared for in family medicine clinics in Mexico. Health Quality Life Outcomes; 8: 20.

2. THAYAPARAN AJ, MAHDI E (2013) The Patient Satisfaction Questionnaire Short Form (PSQ-18) as an adaptable, reliable, and validated tool for use in various settings. Med Educ Online; 18: 21747.

3. ZACHAROVA E (2015) The quality of healthcare from the perspective of the client in prison. Clinical Social Work 1: 70-75.

4. MICHEL R (2015) Satisfaction with the provision of seniors social services. Clinical Social Work; 1: 87-95.

5. HAYS RD, DAVIES RD, WARE JE (1987) Scoring the medical outcomes study patient satisfaction questionnaire: PSQ-III. MOS Memorandum. Santa Monica (CA): Rand Corporation; (unpublished).
6. NGUYEN THI PL, BRIANÇON S, EMPEREUR F, GUILLEMIN F (2002) Factors determining inpatient satisfaction with care. Soc Sci Med; 54: 493-504.

7. CHANDER V, BHARDWAJ AK, RAINA SK, BANSAL P, AGNIHOTRI RK (2011) Scoring the medical outcomes among HIV/ AIDS patients attending antiretroviral therapy center at Zonal Hospital, Hamirpur, using Patient Satisfaction Questionnaire (PSQ18). Indian J Sex Transm Dis; 32: 19-22.

8. HOLIKATTI PC, KAR N, MISHRA A, SHUKLA R, SWAIN SP, KAR S (2012) A study on patient satisfaction with psychiatric services. Indian J Psychiatry 2012; 54: 327-332.

9. EDLUND MJ, YOUNG AS, KUNG FY, SHERBOURNE CD, WELLS KB (2003) Does Satisfaction Reflect the Technical Quality of Mental Healthcare? Health Serv Res; 38: 631-645.

10. MCGLYNN EA (1997) Six challenges in measuring the quality of healthcare. Health Affairs; 16: 7-21.

11. BUTALID L, VERHAAK PF, TROMP F, BENSING JM (2011) Changes in the quality of Dentist-patient communication between 1982 and 2001: an observational study on hypertension care as perceived by patients and general practitioners. BMJ Open; 1: e000203.

12. ĐORĐEVIC V, BRAS M, MILUNOVIC V, BRAJKOVIC L, STEVANOVIC R, POLASEK O (2011) The founding of the Centre for Palliative Medicine, Medical Ethics and Communication Skills: a new step toward the development of patient oriented medicine in Croatia. Croat Med J; 52: 87-88.

13. SIMINOFF LA, STEP MM (2011) A Comprehensive Observational Coding Scheme for Analyzing Instrumental, Affective, and Relational Communication in Healthcare Contexts. J Health Commun; 16: 178-197.

14. JAGADEESAN R, KALYAN DN, LEE P, STINNETT S, CHALLA P (2010) Use of a Standardized Patient Satisfaction 
Questionnaire to Assess the Quality of Care Provided by Ophthalmology Residents. Ophthalmology; 115: 738-743.

15. MCMELLON CA, SCHIFFMAN LG (2001) Cybersenior research: a practical approach to data collection. J Interact Market; 15: 46-55.

16. ESMAEILZADEH P, SAMBASIVAN M, KUMAR N (2010) The Challenges and Issues Regarding E-Health and Health Information Technology Trends in the Healthcare Sector. In Zaman M, Liang Y, Siddiqui SM et al. (ed) E-business Technology and Strategy. pp 23-37. Berlin Heidelberg: Springer-Verlag,.

17. HUGHES R G, ORTIZ E (2005) Medication Errors: Why they happen, and how they can be prevented. Am J Nurs; 105: 14-24.

18. TAYLOR SL, LURIE N (2004) The role of culturally competent communication in reducing ethnic and racial healthcare disparities. Am J Manag Care; 10: SP1-4.

19. NORDYKE RJ, CHANG CH, CHIOU CF, WALLACE JF, YAO B, SCHWARTZBERG LS (2006) Validation of a patient satisfaction questionnaire for anemia treatment, the PSQ-An. Health Qual Life Outcom; 4: 1-10.

20. ZIAEI H, KATIBEH M, ESKANDARI A, MIRZADEH M, RABBANIKHAH Z, JAVADI MA (1982) Determinants of patient satisfaction with ophthalmic services BMC Res Notes 2011;4:1-4.

21. LINN MW, LINN BS, STEIN SR (1982) Satisfaction with ambulatory care and compliance in older patients. Med Care; 20: 606-14.

22. DEWI FD, SUDJANA G, OESMAN YM (2011) Patient satisfaction analysis on service quality of dental healthcare based on empathy and responsiveness. Dent Res J (Isfahan); 8: 172-177. 